\title{
Genetic diagnosis of Duchenne/Becker muscular dystrophy using next-generation sequencing: validation analysis of DMD mutations
}

\author{
Mariko Okubo $^{1,2}$, Narihiro Minami ${ }^{1,3,4}$, Kanako Goto ${ }^{1,3}$, Yuichi Goto ${ }^{3,4}$, Satoru Noguchi ${ }^{1}$, Satomi Mitsuhashi ${ }^{1,3}$ \\ and Ichizo Nishino ${ }^{1,3}$
}

Duchenne and Becker muscular dystrophies (DMD/BMD) are the most common inherited neuromuscular disease. The genetic diagnosis is not easily made because of the large size of the dystrophin gene, complex mutational spectrum and high number of tests patients undergo for diagnosis. Multiplex ligation-dependent probe amplification (MLPA) has been used as the initial diagnostic test of choice. Although MLPA can diagnose $70 \%$ of DMD/BMD patients having deletions/duplications, the remaining $30 \%$ of patients with small mutations require further analysis, such as Sanger sequencing. We applied a high-throughput method using lon Torrent next-generation sequencing technology and diagnosed $92 \%$ of patients with DMD/BMD in a single analysis. We designed a multiplex primer pool for DMD and sequenced 67 cases having different mutations: 37 with deletions/ duplications and $\mathbf{3 0}$ with small mutations or short insertions/deletions in DMD, using an lon PGM sequencer. The results were compared with those from MLPA or Sanger sequencing. All deletions were detected. In contrast, $50 \%$ of duplications were correctly identified compared with the MLPA method. Small insertions in consecutive bases could not be detected. We estimated that lon Torrent sequencing could diagnose $~ 92 \%$ of DMD/BMD patients according to the mutational spectrum of our cohort. Our results clearly indicate that this method is suitable for routine clinical practice providing novel insights into comprehensive genetic information for future molecular therapy.

Journal of Human Genetics (2016) 61, 483-489; doi:10.1038/jhg.2016.7; published online 25 February 2016

\section{INTRODUCTION}

Duchenne muscular dystrophy (DMD; OMIM no.: 310200) is one of the most common neuromuscular diseases of childhood. It is an $\mathrm{X}$-linked recessive disease that affects 1 in 3600-6000 live male births. ${ }^{1,2}$ Becker muscular dystrophy (BMD; OMIM no.: 300376) is milder than DMD. DMD and BMD are caused by mutations in DMD (MIM no. 300377). The dystrophin gene located at Xp21.2 is large and contains 79 exons forming a 14-kb mRNA script with lengthy introns that spans more than $2.5 \mathrm{Mb}$ of the genomic DNA. ${ }^{3}$ The mutational spectrum was reported to be as follows: deletions in $\sim 60 \%$ of patients, duplications in $\sim 10 \%$ of patients and small mutations in $\sim 30 \%$ of patients, including small insertions or deletions within an exon $(\sim 7 \%)$ and nonsense mutations $(\sim 15 \%) .{ }^{4-6}$ Multiplex ligation-dependent probe amplification (MLPA) is a widely used method, and it is the initial diagnostic test of choice in many hospitals. Although MLPA can only diagnose patients with deletions/duplications, another $30 \%$ of patients with point mutations need direct sequencing of all coding regions. Therefore, diagnostic methods have been conducted separately, which eventually increase medical costs and delay diagnosis. ${ }^{7,8}$
At present, promising mutation-specific therapies have been developed. For example, exon skipping is applicable to patients that have a deletion in DMD. In contrast, induction of readthrough of nonsense codons is expected to produce full-length dystrophin in DMD patients with nonsense mutations, which comprise $\sim 15 \%$ of DMD cases. ${ }^{9-12}$ Likewise, in Japan, genetic tests are covered by medical insurance only once in a lifetime. Therefore, a single genetic test that enables a genetic diagnosis in more patients is required.

Recently, next-generation sequencing (NGS) approaches have become major tools for finding the causative genes for diseases. Lim et $a .^{13}$ reported a mutational search platform for the genetic diagnosis of DMD/BMD. Wei et al. ${ }^{14}$ also reported a mutational search for DMD/BMD and female carriers using targeted NGS on HiSeq2000 (Illumina, San Diego, CA, USA). To the best of our knowledge, this is the first study that investigated the clinical availability of Ion Torrent Personal Genome Machine (Ion PGM; Thermo Fisher Scientific, Waltham, MA, USA) for the detection of large deletions, duplications and small mutations in DMD. We selected 67 cases previously diagnosed by MLPA and direct

\footnotetext{
${ }^{1}$ Department of Neuromuscular Research, National Institute of Neuroscience, National Center of Neurology and Psychiatry (NCNP), Tokyo, Japan; ${ }^{2}$ Department of Child Neurology, National Center Hospital, NCNP, Tokyo, Japan; ${ }^{3}$ Department of Genome Medicine Development, Medical Genome Center, NCNP, Tokyo, Japan and ${ }^{4}$ Division of Genetic Diagnosis, Department of Laboratory Medicine, National Center Hospital, NCNP, Tokyo, Japan

Correspondence: Dr S Mitsuhashi, Department of Neuromuscular Research, National Institute of Neuroscience, NCNP; 4-1-1 Ogawa-Higashi, Kodaira, Tokyo 187-8502, Japan. E-mail: smitsuhashi@ncnp.go.jp

Received 16 November 2015; revised 24 December 2015; accepted 15 January 2016; published online 25 February 2016
} 
Table 167 cases with different mutations diagnosed by MLPA or Sanger sequencing

\begin{tabular}{|c|c|c|c|c|}
\hline Patient no & Exon ID & Nucleotide change & Protein change & Mutation \\
\hline 1 & EX25 & c.3408_3409delinsGT & p.GIn $1137^{*}$ & Small insertions \\
\hline 2 & EX3 & c.160_162delCTC & p.Leu54del & Small deletions \\
\hline 3 & EX44 & c.6337 delA & p.lle2113* & Small deletions \\
\hline 4 & EX21 & c.2674delA & p.lle892Phefs*4 & Small deletions \\
\hline 5 & EX53 & c. $7693 \mathrm{C}>\mathrm{T}$ & p.G $\ln 2565^{*}$ & nonsence \\
\hline 6 & EX6 & c. $434 \mathrm{G}>\mathrm{C}$ & p.Arg145Pro & missense \\
\hline 7 & EX69 & c. $10011 C>G$ & p.Cys3337Trp & missense \\
\hline 8 & EX66 & c. $9568 \mathrm{C}>\mathrm{T}$ & p.Arg3190 & nonsence \\
\hline 9 & EX27 & c.3765dupT & p.Gly1256Trpfs* 15 & Small insertions \\
\hline 10 & EX28 & c.3909dupT & p.Glu1304* & Small insertions \\
\hline 11 & int2 & c. $94-1 \mathrm{G}>\mathrm{T}$ & & Splice donor variant \\
\hline 12 & EX59 & c.8821_8822insAGGCCACTTCAAG & p.Asp2944Glyfs*6 & Small insertions \\
\hline 13 & EX68 & c.9816dupT & p.Lys3273* & Small insertions \\
\hline 14 & int3 & c.187-10_187-6delTTGTT & & Splice region variant \\
\hline 15 & EX20 & c.2591delC & p.Ser800Argfs $* 9$ & Small deletions \\
\hline 16 & EX22 & c.2808dupT & p.Asp937* & Small insertions \\
\hline 17 & EX14 & c.1632-15_1639dup52 & p.Thr547llefs*16 & Small insertions \\
\hline 18 & EX21 & c.2673_2674delAAinsG & p.lle892Phefs*4 & Small insertions \\
\hline 19 & EX74 & c.10453_10454delCT & p.Leu3485Glufs*5 & Small deletions \\
\hline 20 & int54 & c. $8218-2 A>G$ & & Splice donor variant \\
\hline 21 & EX20 & c.2430_2443delCCGGTGGATCGAAT & p.Arg811Leufs*6 & Small deletions \\
\hline 22 & EX48 & c.6986delA & p.Lys2329Serfs*9 & Small deletions \\
\hline 23 & EX26 & c.3454_3479del26 & p.Leu 1152 Lysfs*17 & Small deletions \\
\hline 24 & EX48 & c.6923_6933del11 & p.Ala2308Glufs*6 & Small deletions \\
\hline 25 & EX74 & c.10454delT & p.Leu3485Argfs*11 & Small deletions \\
\hline 26 & EX24 & c.3257dupA & p.GIn1087Alafs*11 & Small insertions \\
\hline 27 & EX74 & c.10453dupC & p.Leu3485Prpfs*6 & Small insertions \\
\hline 28 & EX38 & c.5413dupG & p.Val1805Glyfs* 10 & Small insertions \\
\hline 29 & EX15 & c. $1732 \mathrm{~A}>\mathrm{T}$ & p.Lys578* & Nonsence \\
\hline 30 & EX20 & c. $2612 A>C$ & p.Lys871Thr & Missense \\
\hline 31 & EX45 & & & del \\
\hline 32 & EX45-47 & & & del \\
\hline 33 & EX45-48 & & & del \\
\hline 34 & EX45-50 & & & del \\
\hline 35 & EX45-52 & & & del \\
\hline 36 & EX45-55 & & & del \\
\hline 37 & EX48-50 & & & del \\
\hline 38 & EX48-52 & & & del \\
\hline 39 & EX49-50 & & & del \\
\hline 40 & EX51 & & & del \\
\hline 41 & EX45-53 & & & del \\
\hline 42 & EX45-54 & & & del \\
\hline 43 & EX48 & & & del \\
\hline 44 & EX50-52 & & & del \\
\hline 45 & EX2-17 & & & del \\
\hline 46 & EX5-47 & & & del \\
\hline 47 & EX8-28 & & & del \\
\hline 48 & EX2 & & & dup \\
\hline 49 & EX2-7 & & & dup \\
\hline 50 & EX3-7 & & & dup \\
\hline 51 & EX3-13 & & & dup \\
\hline 52 & EX8,9 & & & dup \\
\hline 53 & EX8-11 & & & dup \\
\hline 54 & EX8-17 & & & dup \\
\hline 55 & EX17-19 & & & dup \\
\hline 56 & EX49-50 & & & dup \\
\hline 57 & EX50-55 & & & dup \\
\hline 58 & EX3-9 & & & dup \\
\hline 59 & EX3-30 & & & dup \\
\hline 60 & EX8-41 & & & dup \\
\hline 61 & EX18-48 & & & dup \\
\hline 62 & EX28-55 & & & dup \\
\hline 63 & EX56-67 & & & dup \\
\hline 64 & EX8-29 & & & dup \\
\hline 65 & EX34-44 & & & dup \\
\hline 66 & EX2-6, EX10-18 & & & dup \\
\hline 67 & EX45-53, EX56-60 & & & dup \\
\hline
\end{tabular}

Abbreviations: del, deletion; dup, duplication; MLPA, multiplex ligation-dependent probe amplification. 
sequencing, and developed a novel computational framework for detecting deletions/duplications.

\section{MATERIALS AND METHODS}

\section{Patients}

We chose 67 cases with different mutations to test the clinical utility of our method: 17 cases with common deletions, 20 cases with common duplications ( 2 of them with discontinuous double duplications) and 30 cases with point mutations, including nonsense mutations, deletions, insertions, splice regions and missense mutations (Table 1). All deletion/duplication patterns are frequently diagnosed by MLPA in our laboratory, as shown in Supplementary Tables S1 and S2 and Supplementary Figures S1 and S2.

P3

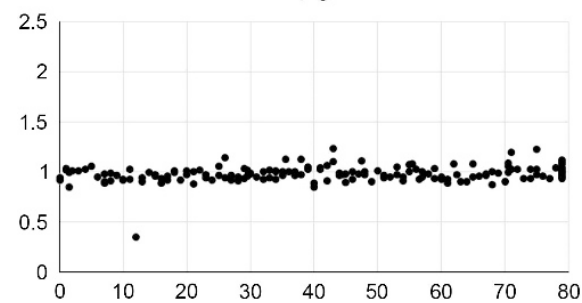

Figure 1 The representative amplicon coverage plot of a control case (P3). The horizontal axis shows exon number and the vertical axis shows the ratio, which is the patient 'r.p.m.' divided by control r.p.m. (r.p.m. $=10^{6} \times(\mathrm{read}$ number for each amplicon)/(read number for each pool exons 70-79)).
Among 30 small mutations, 10 mutations were located within five or more consecutive bases $(\mathrm{P} 16,18,19,22,25,26,27,28,29$ and 30) and 5 mutations were deletion/insertion of more than nine bases (P12, 17, 21, 23 and 24). These mutations were predicted to be difficult to detect by Ion Torrent sequencer. All clinical information and materials used in this study were obtained for diagnostic purposes with written informed consent. This study was approved by the ethics committee of the National Center of Neurology and Psychiatry.

\section{Targeted NGS sequencing}

Targeted NGS covering the dystrophin gene was performed on genomic DNA extracted from blood lymphocytes of patients. Multiplex primer pools were designed using Ion AmpliSeq Designer software (Thermo Fisher Scientific, MA, USA). This custom gene panel covers $99.6 \%$ of the exonic region, including the flanking region (30 base pairs from the exon-intron boundary), and it covers $100 \%$ of the coding region. Enrichment of exonic sequences was performed with an Ion AmpliSeq Library Kit 2.0 (Thermo Fisher Scientific, MA, USA) and sequenced on an Ion PGM (Thermo Fisher Scientific, MA, USA) using 318 Chip (Thermo Fisher Scientific, MA, USA) according to the manufacturer's protocol.

\section{Deletion/duplication detection}

The number of reads mapped on each amplicon was counted by bedtools. ${ }^{15}$ The read that intersects with multiple amplicons was assigned to the amplicon of the largest overlap. The normalized read count was calculated as follows: the raw read count was divided by the total reads on the $\mathrm{C}$-terminal region, which covers exons 70-79, and then multiplied by one million. We used exons 70-79 as we rarely observed mutations in the patients. ${ }^{4-6}$ We used the average value of
P31 EX45del
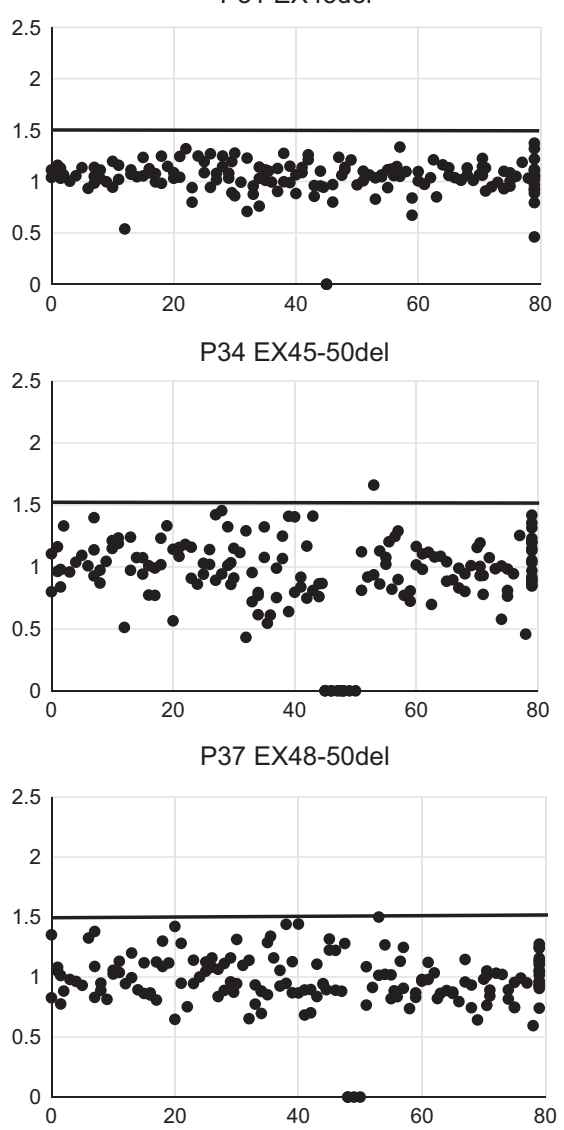

P32 EX45-47del
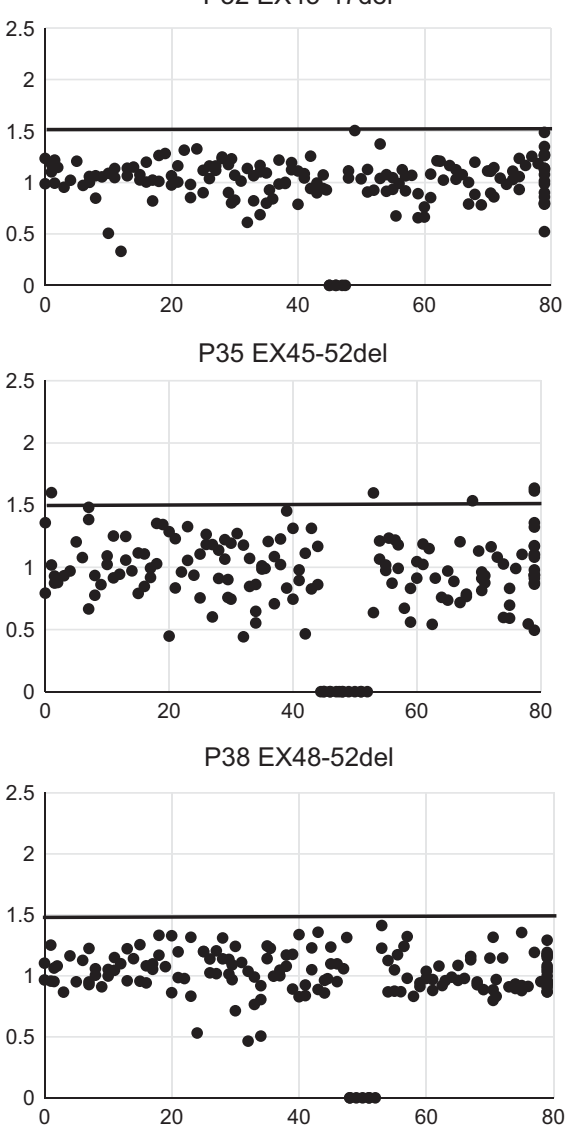
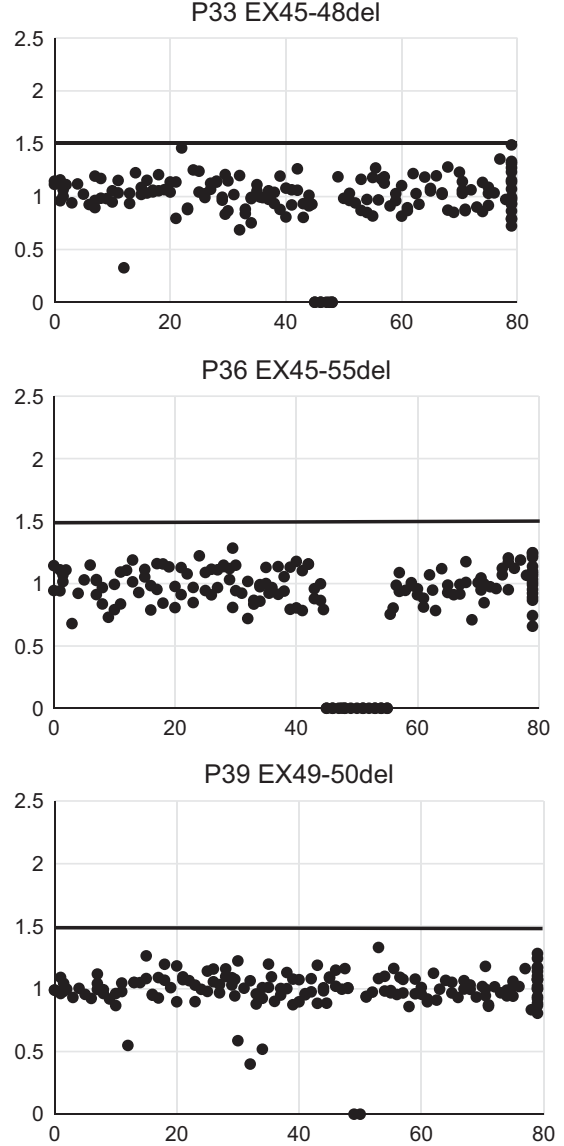

Figure 2 The amplicon coverage plot of 17 patients with large deletion mutation. The horizontal axis shows exon number and the vertical axis shows the ratio, which is the patient r.p.m. divided by control r.p.m. (rpm $=10^{6} \times($ read number for each amplicon)/(read number for each pool exons $\left.70-79)\right)$. Each exon is covered by 1-3 amplicons, only exon 79 is covered by 13 amplicons. If the ratio is zero, the patient has a deleted exon. 

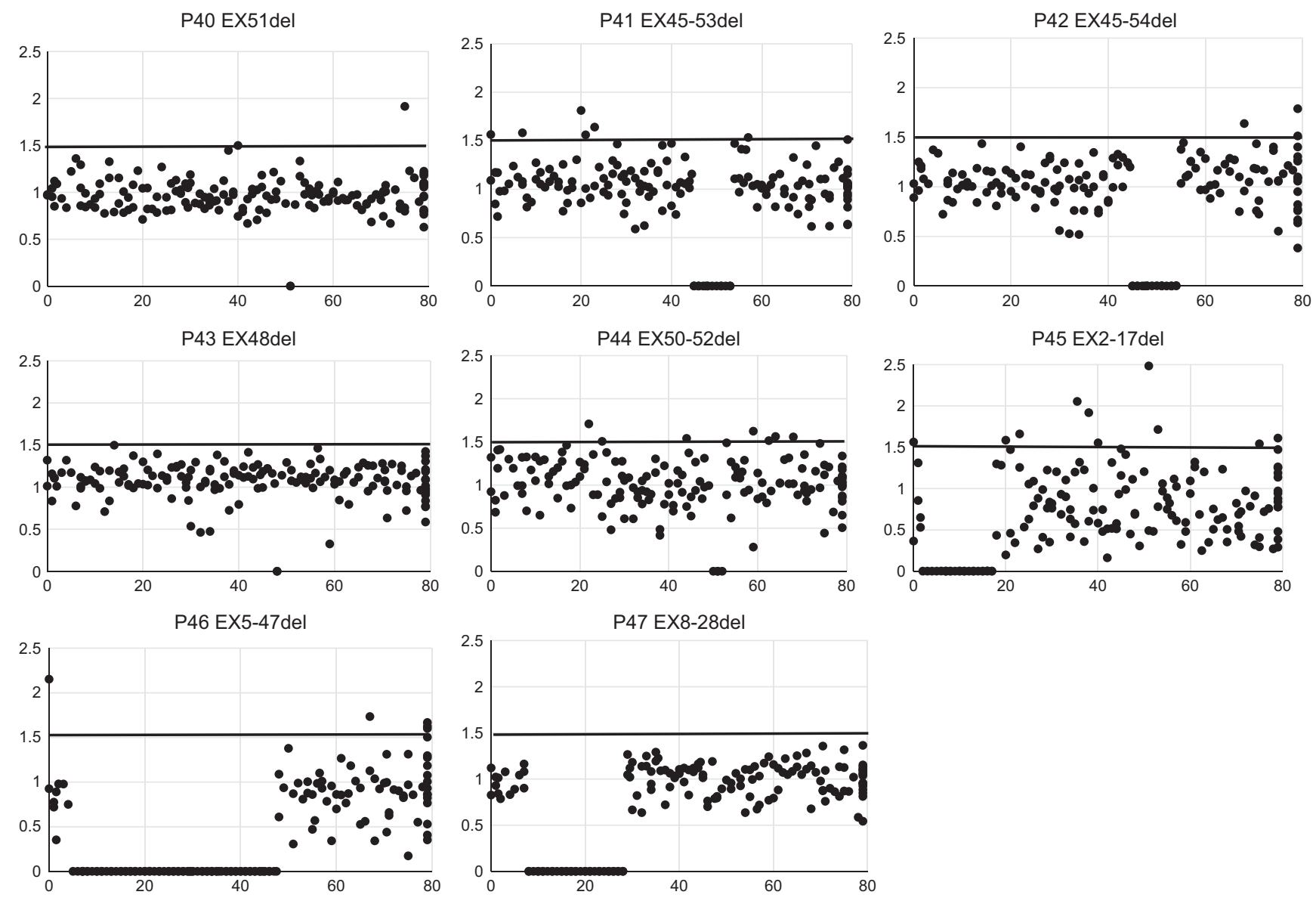

Figure 2 Continued

the 19 control cases. For each amplicon, the mean value and sample s.d. were calculated. It was considered to be duplicated when both of the following conditions were met: if the normalized read count was $>1.5$-fold of the mean value in control samples and if the normalized read count deviated from the mean value by over 2 -fold of sample s.d., the amplicon (Supplementary Figure S3).

\section{RESULTS}

Most small mutations were detected by targeted NGS (P1-30)

We chose 15 cases with different small mutations, together with 15 mutations predicted to be difficult to detect by Ion Torrent sequencing, such as single-nucleotide variants ( $\mathrm{SNVs}$ ) in the same consecutive bases or relatively large deletions. We failed to detect five small insertions. Among these five, there were four small insertions (P17, 18, 26 and 27) that were located in the same consecutive bases.

Control cases for copy number analysis (P1-19)

To set a normalized read count, we performed amplicon coverage on 19 cases (P1-19) that were negative for deletion/duplication mutation in $D M D$ by MLPA analysis (Figure 1). The coverage ratio for all amplicons was $1 \pm 0.5$. The amplicon for exon 12 had low amplification on 19 cases, leading to a ratio of 1.5 that was not usable. The cutoff values for the 19 controls were 2 times the s.d. and 1.5 times for the duplication (Supplementary Figure S3).
All large deletions were detected by targeted NGS (P31-47)

The coverage plot of the 17 large deletion cases is shown in Figure 2. For deletion cases (P31-47), the deleted exons detected by NGS were completely consistent with the results obtained using MLPA. There was a variation in the ratio of each amplicon for P45.

Most large duplications were detected by targeted NGS (P48-67) The coverage plot of the 20 large duplication cases is shown in Figure 3. In the cases with large duplications (P48-67), 10 of the 20 duplicated exon cases were accurately detected (P48, 51, 54, 55, 56, 58, 63, 64, 66 and 67). Ten cases (P49, 50, 52, 53, 57, 59, 60, 61, 62 and 65) had false negative or positive amplicons. However, seven cases (P49, 50, 57, 59, 60, 62 and 65) had only one false negative or positive amplicon. Therefore, overall, we can detect the precise duplicated exons in these seven cases. P52 had several false positive regions due to marked variation in read counts; thus we could not identify the duplicated exon.

P53 was reported to have exons 8-11 duplication by MLPA, but the Ion PGM result showed that exons 8, 10 and 11 were duplicated and exon 9 was deleted. We suspected that Ion PGM primers failed to amplify the exon 9 amplicon due to a nucleotide variant in the primerbinding site. Thus, we sequenced exon 9 and identified point mutations at a primer site in the exon 9-flanking region in intron 8 (c.832-53C $>$ T).

MLPA showed that P61 has an exons 18-48 duplication, but the Ion PGM result showed that exons 18-47 were duplicated. We also suspected that there are variants that may interfere with the MLPA 
probe or Ion PGM primers. We sequenced exon 48 by Sanger sequencing but found no small mutations in exon 48 .

\section{DISCUSSION}

In this study, we established the clinical utility of Ion Torrent sequencing technology for the detection of deletions/duplications and small mutations in DMD by comparison with the conventional method, MLPA and Sanger sequencing in the large number of the $\mathrm{DMD} / \mathrm{BMD}$ patients in the Japanese cohort. As we expected, all large deletions and $50 \%$ of the large duplications are correctly detected. We also aimed to clarify the percentage of the detection yield of the small mutations predicted to be difficult to read by Ion PGM. Three out of 10 mutations located with $>5$ consecutive bases and one out of five small deletions/insertions of more than 10 bases were not detected. Out of 255 patients with point mutations in our in-house data (Supplementary Figure S4), we estimate that Ion PGM could not identify $1 \%$ of DMD/BMD cases with such small insertions (Figure 4).

Previous reports have demonstrated the ability of NGS, Illumina sequencer to detect SNVs and deletions/duplications in $D M D^{13}$ and copy number variations (CNVs) in female carriers. ${ }^{14}$ Gene panels that contained many genes (Lim et al. ${ }^{13}$ with 25 genes and Wei et al. ${ }^{14}$ with 222 genes), including $D M D$, were used in these studies. In this report, we targeted single DMD gene using Ion PGM for the first time that effectively enables us to perform the test at a lower cost compared that with other studies. Because of the small sample size in the previous study and different cohort of the mutation tested, we do not know which sequencer has more advantage on DMD gene testing. Further comparison study will be necessary.

We used multiplex primer pools covering 100\% of the coding region, comprising 161 amplicons for 79 exons. One to four amplicons covered each exon except exon 79, which was covered by 13 amplicons. When an exon is covered only by one amplicon, it would be difficult to determine when there is a singlenucleotide polymorphism at the primer site. For example, P53 showed an exon 9 deletion by Ion PGM, but we could amplify exon 9 by PCR and Sanger sequencing identified with a point mutation at the primer site of Ion PGM. In P48 and P56, we detected duplication of exon 2 and 49-50, respectively, but these exons are covered by one amplicon. Therefore, it should be noted that if there were a point mutation at the primer site, we could have not correctly diagnosed these cases. This problem can also occur with MLPA when it detects a single exon deletion. If there is a sequence variation close to the probe ligation site deletion/duplication will not be identified correctly by MLPA. ${ }^{16}$ As for the Ion PGM, we recommend designing two or more amplicons for each exon to increase the diagnostic accuracy.
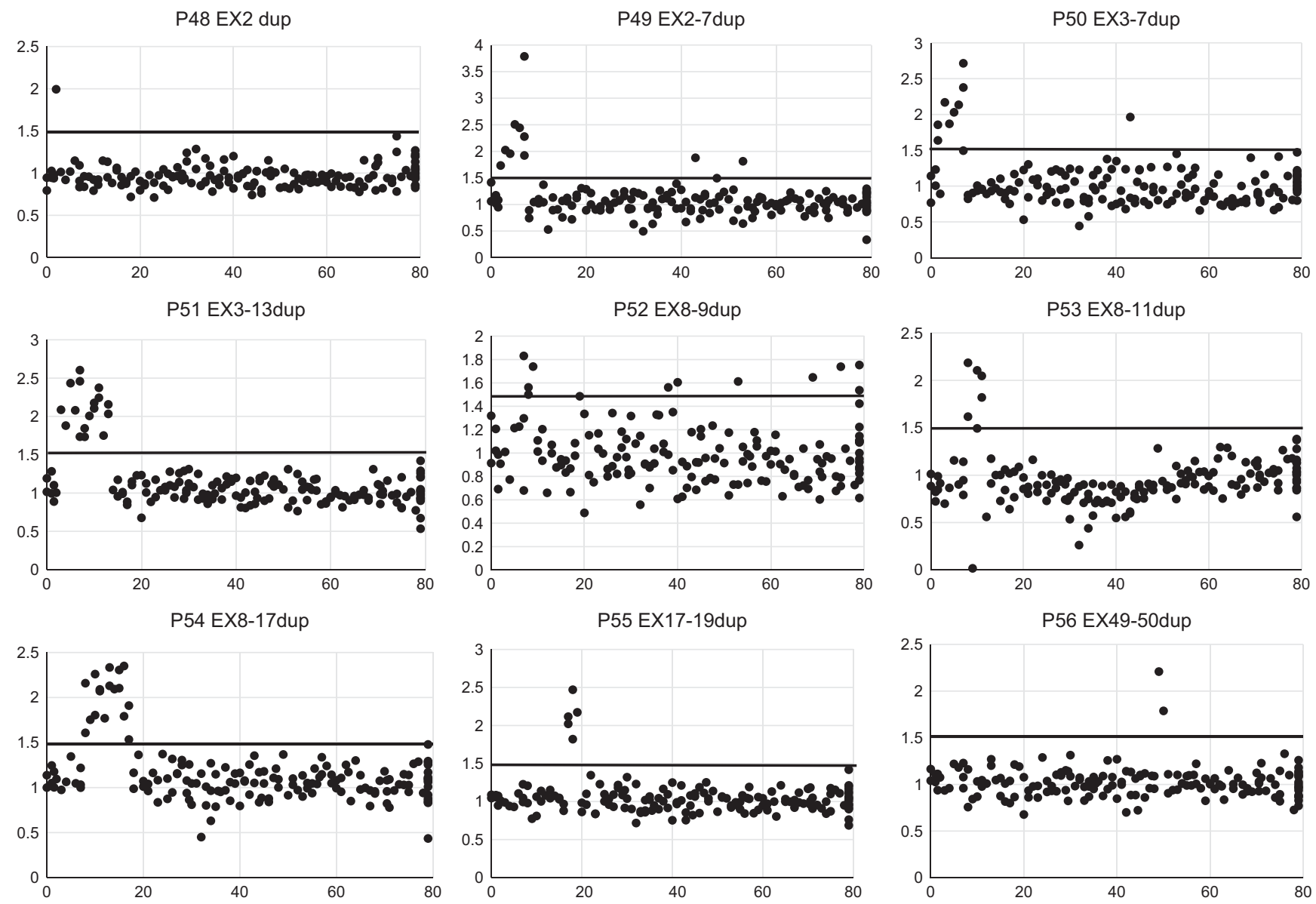

Figure 3 The amplicon coverage plot of 20 patients. The horizontal axis shows the exon number and the vertical axis shows the ratio, which is the patient $r$. p.m. divided by control r.p.m. ( $r p m=10^{6} \times($ read number for each amplicon)/(read number for each pool exons $70-79)$ ). If the value of the ratio is 1.5 or more, the patient has a supplicated exon. We have drawn a line at the ratio of 1.5 . 

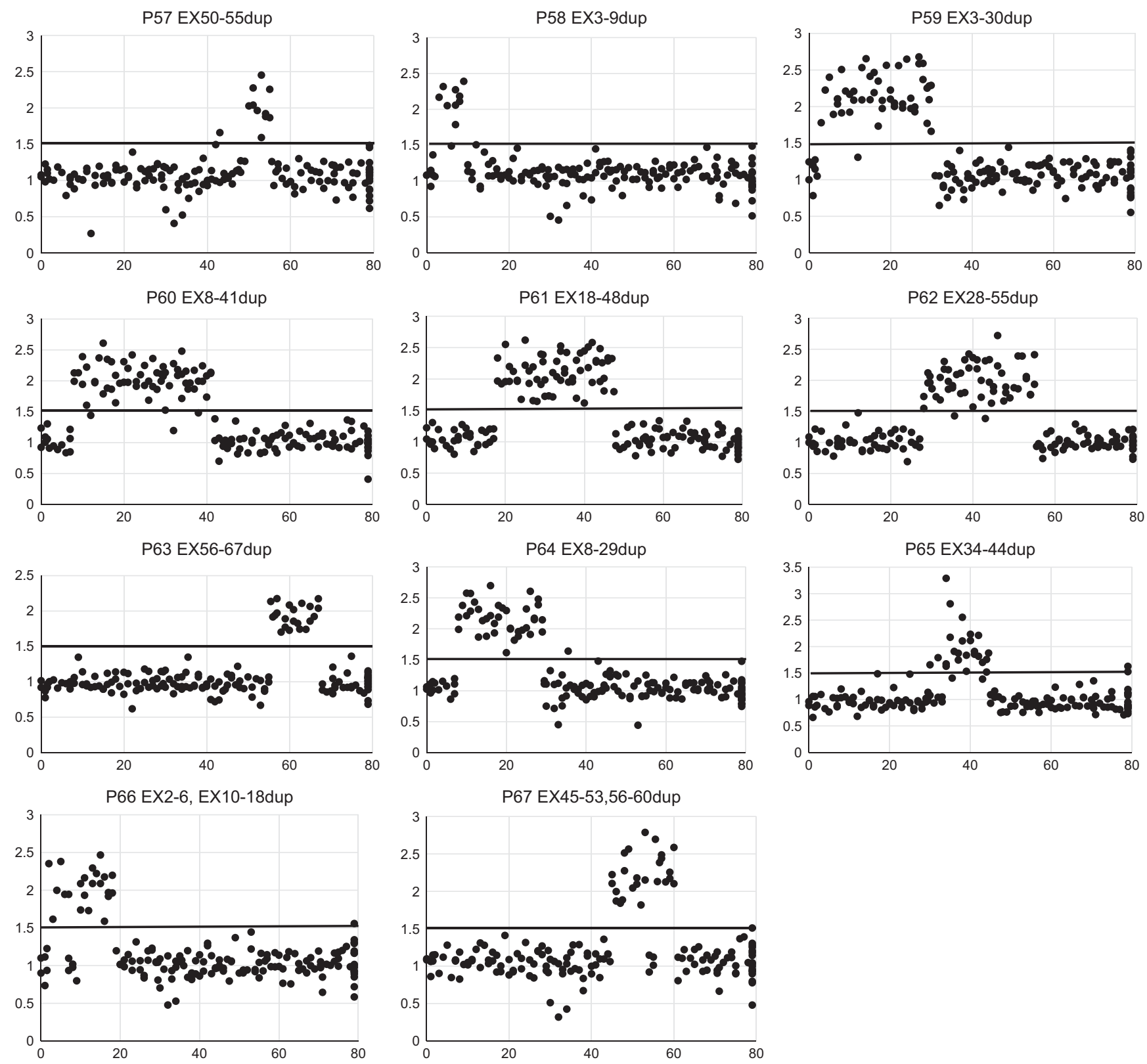

Figure 3 Continued

Detecting the large duplications has some difficulty in both MLPA and Ion PGM. For example, MLPA showed that P61 has an exons 18-48 duplication, but the Ion PGM result showed that exons 18-47 was duplicated. In this study, exon 48 was covered by two amplicons. If there is a breakpoint within the exon 48 that disrupts the binding of either PCR primer, but preserves the MLPA probe site, it is possible to have a confounding result between MLPA and coverage analysis (Supplementary Figure S5). This could occur in detecting duplications by both MLPA and Ion PGM. Thus, we require careful interpretations for detecting the end regions of exons for putative duplication events.

Given the difficulty in interpreting certain large duplications and insertions, MLPA or Sanger sequencing may be required to complement Ion PGM in some cases. Nonetheless, a single genetic test that could give a precise genetic diagnosis to more patients would be preferable, saving time and money for the patients. Accordingly, we estimate that $92 \%$ of DMD/BMD patients are accurately diagnosed using the Ion PGM (Figure 4). In addition, we assume that sequencing $D M D$ gene even in the patients with copy-number variation mutation will be necessary to exclude the possibility of a rare single-nucleotide polymorphism that might impact exon skipping therapy or possible double mutation, which can blemish the effect of gene therapy. Therefore, we believe NGS will be a standard for the diagnosis of $\mathrm{DMD} / \mathrm{BMD}$ in the future.

Here, we suggest a new diagnostic flowchart for DMD/BMD patients in the future. First, if the patient is suspected to have $\mathrm{DMD} / \mathrm{BMD}$, we recommend performing Ion PGM for DMD $(92 \%$ are estimated to be diagnosed). In addition, MLPA can work as a complimentary test to confirm the duplication that is difficult to interpret from Ion PGM result (5\%). If the mutation is not detected, 


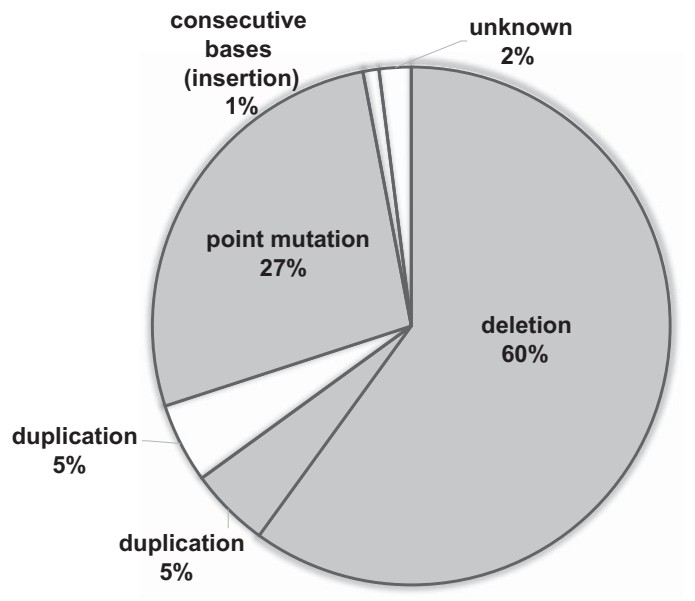

Figure 4 The mutational spectrum from our in-house data and reported data.

the next step is to examine dystrophin expression on the sarcolemma and exclude a possibility of loss of other proteins such as sarcoglycans in the muscle biopsy specimen, and to perform direct Sanger sequencing for all coding regions to detect mutations that are difficult to read by Ion PGM. This proposed diagnostic flowchart will finally give a genetic diagnosis for $98 \%$ of patients. Our in-house data showed there are $2 \%$ of the patients in whom we could not detect any mutations by MLPA or Sanger sequencing of all the exons and flanking regions, but show abnormal dystrophin staining. These patients might have mutations in deep introns or dystrophin expression abnormality might be secondary. Therefore, we demonstrate that NGS can be used for an initial genetic diagnosis test of DMD/BMD.

This is the first study to show the utility of Ion PGM to detect both deletions/duplications and small mutations in DMD/BMD patients.

\section{CONFLICT OF INTEREST}

The authors declare no conflict of interest.

\section{ACKNOWLEDGEMENTS}

This study was supported partly by Intramural Research Grant $(26-8,26-7)$ for Neurological and Psychiatric Disorders of NCNP, and Health and Labour Sciences Research Grants for Practical Research Project for Rare/Intractable Diseases (H26-Itaku (Nan)-Ippan-081) from Japan Agency for Medical Research and Development.
1 Emery, A. E. Population frequencies of inherited neuromuscular disease-a world survey Neuromuscl. Disord. 1, 19-29 (1991).

2 Bushby, K., Finkel, R., Brinkrant, D. J., Case, L. E., Clemens, P. R., Cripe, L. et al. Diagnosis and management of Duchenne muscular dystrophy, part1: diagnosis, and pharmacological and psychosocial management. Lancet Neurol. 9, 77-93 (2010).

3 Ahn, A. H. \& Kunkel, L. M. The structural and functional diversity of dystrophin. Nat. Genet. 3, 283-291 (1993).

4 Takeshima, Y., Yagi, M., Okizuka, Y., Awano, H., Zhang, Z., Yamauchi, Y. et al. Mutation spectrum of dystrophin gene in 442 Duchenne/Becker muscular dystrophy cases from one Japanese referral center. J. Hum. Genet. 55, 379-388 (2010).

5 Magri, F., Govoni, A., Angelo, M. G., Del Bo, R., Gbezzi, S., Sandra, G. et al. Genetype and phenotype characterization in a large dystrophinopathic cohort with extended follow-up. J. Neurol. 258, 1610-1623 (2011).

6 Mah, J. K., Selby, K., Campbell, C., Nadeau, A., Tarnopolsky, M., McCormicj, A. et al. A Population-Based Study of Dystrophin Mutation in Canada. Can. J. Neurol. Sci. 38, 465-474 (2011).

7 Lalic, T., Vossen, R. H., Coffa, J., Schouten, J. P., Guc-Scekic, M., Radivojevic, D. et al Deletion and duplication screening in the DMD gene using MLPA. Eur. J. Hum. Genet. 13, 1231-1234 (2005).

8 Flanigan, K. M., Dunn, D. M., Niederhausem, A. V., Soltanzadeh, P., Gappmarie, E. Howard, M. T. et al. Mutational spectrum of dmd mutations in dystrophinopathy patients: application of modern diagnostic techniques to a large cohort. Hum. Mutat. 30, 1657-1666 (2009).

9 MaliK, V., Rodino-Klapac, L. R., Viollet, L., Wall, C., King, W., Al-Dahhak, R. et al. Gentamicin-Induced readthrough of stop codons in duchenne muscular dystrophy. Ann. Neurol. 67, 771-780 (2010).

10 Welch, E. M., Barton, E. R., Zhuo, J. Tomizawa, Y., Friesen, W. J., Trifillis, P. et al. PTC124 targets genetic disorders caused by nonsense mutations. Nature 447, 87-93 (2007).

11 Finkel, R. S., Flanigan, K. M., Wong, B., Bonnemann, C., Sampson, K., Sweeney, H. L. et al. Phase $2 \mathrm{a}$ study of ataluren-mediated dystrophin production in patients with nonsense mutation duchenne muscular dystrophy. PLOS ONE 8, e81302 (2013).

12 Falzarano, M. S., Scotton, C., Passarelli, C. \&, Ferlini, A. Duchenne muscular dystrophy: from diagnosis to therapy. Molecules 28, 18168-18184 (2015).

13 Lim, B. C., Lee, S., Shin, J. Y., Kim, J. I., Hwang, H., Kim, K. J. et al. Genetic diagnosis of Duchenne and Becker muscular dystrophy using next-generation sequencing technology: comprehensive mutational search in a signal platform. J. Med. Genet. 48, 731-736 (2011).

14 Wei, X., Dai, Y., Yu, P., Qu, N., Lan, Z., Hong, X. et al. Targeted next-generation sequencing as a comprehensive test for patients with and female carriers of DMD/BMD: a multi-population diagnostic study. Eur. J. Hum. Genet. 22, 110-118 (2014).

15 Quinlan, A. R. \& Hall, I. M. BEDTools: a flexible suite of utilities for comparing genomic features. Bioinformatics 26, 841-842 (2010).

16 Janssen, B., Hartmann, C., Scholz, V., Jauch, A. \& Zschocke, J. MLPA analysis for the detection of deletions, duplications and complex rearrangements in the dystrophin gene: potential and pitfall. Neurogenetics 6, 29-35 (2005).

This work is licensed under a Creative Commons Attribution-NonCommercial-NoDerivs $\quad 4.0$ International License. The images or other third party material in this article are included in the article's Creative Commons license, unless indicated otherwise in the credit line; if the material is not included under the Creative Commons license, users will need to obtain permission from the license holder to reproduce the material. To view a copy of this license, visit http://creativecommons.org/licenses/bync-nd/4.0/

Supplementary Information accompanies the paper on Journal of Human Genetics website (http://www.nature.com/jhg) 